\title{
Expression and Pathogenic Analysis of Integrin Family Genes in Systemic Sclerosis
}

\author{
Dan $\mathrm{Xu}^{1 \dagger}$, Ting $\mathrm{Li}^{1 \dagger}$, Ruikang Wang ${ }^{2}$ and Rong $\mathrm{Mu}^{1 *}$ \\ 1 Department of Rheumatology and Immunology, Peking University Third Hospital, Beijing, China, ${ }^{2}$ Department of \\ Rheumatology and Immunology, Peking University People's Hospital, Beijing, China
}

Objectives: Emerging evidence shows that integrin members are involved in inflammation and fibrosis in systemic sclerosis (SSc). This study aimed at evaluating the expression of integrin family genes in the skin tissue from SSc patients and exploring the potential pathogenic mechanism.

Methods: We utilized the public datasets of SSc skin tissue from the Gene Expression Omnibus (GEO) database to analyze the expression and clinical significance of integrin family genes in SSc. The expression of integrin members in skin tissue was also assessed by immunohistochemistry. In addition, functional enrichment and pathway analysis were conducted.

OPEN ACCESS

Edited by:

Isotta Chimenti,

Sapienza University of Rome, Italy

Reviewed by:

Roberto Giacomelli,

University of L'Aquila, Italy

Heiko Golpon,

Hannover Medical School, Germany

*Correspondence:

Rong Mu

murongster@gmail.com

${ }^{\dagger}$ These authors have contributed equally to this work

\footnotetext{
Specialty section:

This article was submitted to

Pathology,

a section of the journal

Frontiers in Medicine
}

Received: 01 March 2021

Accepted: 17 June 2021

Published: 20 July 2021

Citation:

Xu D, Li T, Wang R and Mu R (2021) Expression and Pathogenic Analysis of Integrin Family Genes in Systemic Sclerosis. Front. Med. 8:674523. doi: 10.3389/fmed.2021.674523
Results: Compared with healthy controls, the mRNA and protein levels of ITGA5, ITGB2, and ITGB5 were upregulated in the skin of SSc patients. Further analysis indicated that the mRNA expression levels of ITGA5, ITGB2, and ITGB5 were positively correlated with modified Rodnan skin thickness score (mRSS). Functional enrichment and pathway analysis showed that integrin members may play multiple roles in the pathogenesis of SSc. Among them, ITGA5, ITGB2, and ITGB5 might synergistically promote SSc through affecting extracellular matrix (ECM) turnover, ECM-receptor interaction, focal adhesion, and leukocyte trans-endothelial migration, while ITGA5 and ITGB5 also might affect angiogenesis and endothelial cell function. In addition, ITGA5, ITGB2, and ITGA5 were associated with different pathways, respectively. ITGA5 was uniquely enriched for actin organization, while ITGB5 was for TGF- $\beta$ signaling and ITGB2 for immune cell activation.

Conclusion: Our results implied that the abnormal expression of integrin family genes including ITGA5, ITGB2, and ITGB5 may participate in multiple pathological processes in SSc. Further investigations are required for confirming this speculation.

Keywords: integrin, systemic sclerosis, skin, bioinformatics analysis, gene

\section{INTRODUCTION}

Systemic sclerosis (SSc) is an immune-mediated autoimmune disease, which is characterized by activation of the immune system, dysfunction of endothelial cells, and organ fibrosis $(1,2)$. The aberrant accumulation of extracellular matrix (ECM) components often disrupts the physiological architecture and leads to angiogenesis and organ fibrosis (3). It has been implied that the interaction between the surrounding ECM, fibroblasts, and endothelial cells is mediated by the expression of specific integrins on the cell surface, which strongly affects cell function (4). 
Integrins are a large family of heterodimeric transmembrane receptors, comprising $\alpha$ and $\beta$ subunits. There are $18 \alpha$ subunit genes (ITGA1, ITGA2, ITGA3, ITGA4, ITGA5, ITGA6, ITGA7, ITGA8, ITGA9, ITGA10, ITGA11, ITGA2B, ITGAD, ITGAE, ITGAL, ITGAM, ITGAV, ITGAX) and $8 \beta$ subunit genes (ITGB1, ITGB2, ITGB3, ITGB4, ITGB5, ITGB6, ITGB7, $I T G B 8$ ), forming 24 different transmembrane surface receptors in vertebrates (5). Integrins are widely distributed on cell surfaces of normal fibroblast, endothelial cells, epithelial cells, and immune cells. Integrin changes conformation and activates subsequent intracellular signals by recognizing specific extracellular ligands, so as to mediate cell-cell adhesion and cell-matrix adhesion, respond to microenvironment signals, and regulate cell migration and proliferation (6). Emerging evidence shows that integrins also play important roles in autoimmunity (7), angiogenesis (8), and tissue fibrosis (9) by controlling local activation of latent transforming growth factor (TGF)- $\beta$, cross-talking with growth factor receptors (10), mediating mechanotransduction (11), and promoting cell adhesion and proliferation (12).

ITGA5, ITGB1, ITGB3, and ITGB5 are well-studied integrin members in SSc, which can form $\alpha 5 \beta 1, \alpha v \beta 3$, and $\alpha v \beta 5$ heterodimeric receptors. Among them, integrin $\alpha \mathrm{V} \beta 3$ and integrin $\alpha \mathrm{V} \beta 5$ were found overexpressed in SSc patients and animal models, which were related to lymphocyte infiltration, TGF- $\beta$ activation, and collagen accumulation (13). On the other hand, studies on other integrin members still remain sparse. Gene analysis revealed that inflammation mediated by the integrin signaling pathway was a common shared pathway related to SSc (14). All these discoveries made integrin an attractive therapeutic target for SSc. Neutralizing antibody and small molecule inhibitors of integrin $\alpha \mathrm{V}$ and $\beta 1$ were protective in animal models of SSc $(13,15,16)$. However, integrin-targeted therapy has not shown significant efficacy in patients yet. Phase II clinical trials of an $\alpha \mathrm{V} \beta 6$ antibody (BG00011) in the treatment of idiopathic pulmonary fibrosis (IPF) and an integrin $\alpha \mathrm{V}$ monoclonal antibody (abituzumab) in the treatment of SScILD were terminated due to safety and efficacy problems or difficulties in patient inclusion, respectively (clinicaltrials.gov identifier NCT03573505 and NCT02745145). The failure of the above clinical trials may be partly due to the complexity of the integrins in the progression of SSc. This study aimed to explore the key members and roles of integrins in the pathogenesis of SSc, therefore providing insight into its potential value as a therapeutic target for SSc.

\section{METHODS}

\section{Microarray Data Acquisition and Processing}

To explore the changes of expression profiling and reveal biological processes in the skin of patients with SSc, eligible microarray datasets were downloaded from the Gene Expression Omnibus (GEO, http://www.ncbi.nlm.nih.gov/geo) database. The inclusion criteria of microarray datasets were as follows: (1) datasets should be the expression profile of genome-wide mRNA transcriptome data, (2) datasets consisted of expression profiles in the skin of patients with SSc and normal controls, and (3) datasets were standardized or raw datasets. All gene expression data have previously been published on the GEO database.

\section{Identification of Differentially Expressed Integrin Family Genes}

Normalization and $\log 2$ transformation were performed for the raw data to minimize heterogeneity. Probes without gene symbols or genes with more than one probe were removed or averaged, respectively. The merged data were preprocessed by sav package to remove batch effects with $\mathrm{R}$ software (version 3.5.1). After performing batch normalization, limma package was utilized to screen differentially expressed integrin family member genes between SSc patients and healthy controls. Adjusted $p<0.05$ was considered statistically significant. The correlations between mRNA expression of integrin members and clinical characteristics were analyzed.

\section{Immunohistochemistry (IHC)}

Immunohistochemical analysis was performed on 3-mm sections of formalin-fixed, paraffin-embedded tissue. Antigen retrieval was performed by a microwave oven with buffer of citric acid ( $\mathrm{pH}$ 6.0). Then, endogenous peroxidase activity and nonspecific binding were blocked with peroxidase block buffer and $1 \%$ bovine serum albumin, respectively. After blocking, sections were incubated with primary antibodies (anti-integrin a5 antibody, 1:400, ab150361, Abcam, Cambridge, MA, USA; anti-integrin $\alpha 7$ antibody, 1:800, ab203254, Abcam, Cambridge, MA, USA; anti-integrin $\beta 2$ antibody, 1:2,000, ab131044, Abcam, Cambridge, MA, USA; anti-integrin $\beta 5$ antibody, 1:400, 3629S, CST, Danvers, MA, USA) at $4^{\circ} \mathrm{C}$ overnight. Subsequently, sections were incubated with HRP conjugate before chromogenic detection using $\mathrm{DAB}$. For the semiquantitative analysis, the $\mathrm{H}-$ score method assigned a score of $0-300$ to each patient, based on the percentage of cells stained at different intensities (17).

\section{GO and KEGG Enrichment Analyses}

Database for Annotation, Visualization, and Integrated Discovery (DAVID) was applied to perform the Gene Ontology (GO) analysis and Kyoto Encyclopedia of Genes and Genomes (KEGG) pathway enrichment analysis. The integrin family genes showed correlation coefficient $(r)>0.5$ were analyzed for GO and KEGG enrichment by DAVID. GO analysis included biological processes (BP), cellular components (CC), and molecular functions (MF). The top 10 significant enrichments of $\mathrm{BP}, \mathrm{CC}$, and MF ( $p<0.05$ and rank by $p$-value) and KEGG enrichments $(p<0.05)$ were visualized.

\section{Estimating the Population Abundance of Tissue-Infiltrating Immune and Stromal Cell Populations}

The absolute abundance of eight immune and two stromal cell populations were analyzed by Microenvironment Cell Populations-counter (MCP-counter) method (18). In addition, we analyzed the association of integrin members and the 
TABLE 1 | Studies included in the analysis ${ }^{a}$.

\begin{tabular}{llccc}
\hline Dataset & $\begin{array}{l}\text { GEO } \\
\text { accession } \\
\text { no. }\end{array}$ & $\begin{array}{c}\text { Excluded, } \\
\text { no. }\end{array}$ & $\begin{array}{c}\text { Included, } \\
\text { no. }\end{array}$ & $\begin{array}{c}\text { No. of } \\
\text { samples }\end{array}$ \\
\hline Assassi et al. (19) & GSE58095 & 5 & 97 & $\begin{array}{c}61 \mathrm{SSc}, 36 \\
\mathrm{HC}\end{array}$ \\
Guo et al. (20) & GSE65336 & 68 & 10 & $\begin{array}{c}10 \mathrm{SSC} \\
\text { Mantero et al.* }\end{array}$ \\
GSE95065 & 0 & 33 & $\begin{array}{c}18 \mathrm{SSc}, 15 \\
\mathrm{HC}\end{array}$
\end{tabular}

Eighty-nine SSc patients and $61 \mathrm{HC}$ were included.

SSc, systemic sclerosis; HC, healthy control.

${ }^{a}$ Arrays were excluded if the samples were not skin biopsies or if the samples were collected at a second time point.

*Array profiling data can be obtained from the Gene Expression Omnibus (GEO-NCBI) (GSE95065), which is shared by Mantero JCP. et al. The website is https://www.ncbi. nlm.nih.gov/geo/query/acc.cgi?acc=GSE95065.

population abundance of tissue-infiltrating immune and stromal cell by Spearman's correlation.

\section{RESULTS}

\section{Gene Expression Datasets of SSc}

Three expression datasets were obtained with accession numbers of GSE58095, GSE65536, and GSE95065 from the GEO database $(19,20)$. Totally, skin gene expression profiles of 89 SSc patients and 61 normal controls were enrolled in this study. The detailed information of the four datasets is displayed in Table $\mathbf{1 .}$

\section{The Aberrant mRNA Expression of Integrin Genes in SSc Patients}

To understand the roles of integrin genes in SSc, we compared the mRNA levels of integrin genes between SSc and normal controls. The results indicated that mRNA levels of ITGA5, ITGA7, ITGA8, $I T G B 2$, and ITGB5 were significantly higher in skin samples of SSc than those of normal controls, while the mRNA levels of $I T G A E$ and $I T G B 3 B P$ were significantly lower and other integrins showed no remarkable differences (Figure 1).

\section{The Protein Levels of Abnormal Integrins in the Skin Tissues of SSc Patients}

We next performed an immunohistochemical analysis to evaluate the expression of integrin $\alpha 5$, integrin $\alpha 7$, integrin $\beta 2$, and integrin $\beta 5$ in the skin tissue from 10 SSc patients and 6 normal controls. Consistent with the results of gene expression profiles from microarray, the protein levels of integrin $\alpha 5$, integrin $\beta 2$, and integrin $\beta 5$ in the skin tissues of SSc patients were significantly increased compared with those in the normal control, respectively. However, the level of integrin $\alpha 7$ was found to have no significant differences between the skin tissues of normal and SSc patients (Figure 2).

\section{Association Between Aberrant Expressed Integrin Family Genes and Clinical Features of SSC}

To verify the potential roles of aberrant expressed integrin family genes in SSc, correlation analysis and subgroup analysis between the mRNA levels of ITGA5, ITGB2, and ITGB5 and clinical features were performed in GSE58095. The clinical characteristics of SSc subjects from GSE58095 are concluded in Table 2. We found that the increased ITGA5, ITGB2, and ITGB5 expressions were significantly associated with modified Rodnan skin thickness score (mRSS) (Figure 3A). Concerning the SSc subgroups, the mRNA expression levels of ITGA5 and ITGB5 were higher in dcSSc skin tissue than in lcSSc (Figure 3B). We compared integrin family gene expression in the skin sample according to the type of autoantibody in SSc patients, while we observed that ITGB2 was downregulated in patients seropositive for anti-centromere antibody (ACA) compared with those lacking specific antibodies. We also found that the gene expression of ITGB5 was higher in patients seropositive for ACA than in those lacking specific antibodies or those seropositive for either anti-U1 RNP antibody (RNP) or anti-topoisomerase antibody (ATA) (Figure 3C).

\section{Correlation Between Aberrant Expressed Integrin Family Genes and the Infiltration of Immune and Stromal Cells in the Skin of SSc}

The composition of immune cells, fibroblast, and endothelial cell in the skin of SSc patients and healthy control was analyzed, and the fraction of eight kinds of immune cells was shown in the violin plot (Figures $4 \mathbf{A}-\mathbf{J}$ ). As shown, the abundance of monocytic lineage, endothelial cell, and fibroblast was higher in SSc patients than in healthy controls, while the abundance of $\mathrm{CD}^{+} \mathrm{T}$ cell was relatively lower in SSc patients.

The mRNA expression of ITGA5, ITGB2, and ITGB5 showed significant correlation with the abundance of tissue-infiltrating immune cells or stromal cells in the SSc skin sample. Among them, the mRNA expression level of ITGA5 was positively correlated with the abundance of fibroblasts $(r=0.47, p<$ $0.0001)$, endothelial cells $(r=0.44, p<0.0001)$, and monocytes $(r=0.35, p<0.0001)$, suggesting that ITGA5 may participate in the pathogenesis of scleroderma through these cells. The mRNA expression level of ITGB2 was significantly correlated with the abundance of fibroblasts $(r=0.64, p<0.0001)$, endothelial cells $(r=0.52, p<0.0001)$, T cells $(r=0.36, p<0.0001)$, and myeloid dendritic cells $(r=0.39, p<0.0001)$. The mRNA expression level of ITGB5 was positively correlated with the abundance of fibroblasts $(r=0.63, p<0.0001)$, endothelial cells $(r=0.43, p$ $<0.0001)$, and monocytes $(r=0.26, p=0.0021)$. The detailed correlation between the abundance of immune and stromal cell types and mRNA expression levels of integrin member genes is shown in Figure 4K.

\section{Possible Mechanisms of ITGA5, ITGB2, and TIGB5 Involved in SSC}

To explore the potential function of ITGA5, ITGB2, and ITGB5 in SSc, their co-expressed genes $(r>0.5)$ were analyzed by GO and KEGG in the DAVID database. There were the same GO terms and KEGG pathway that appeared in ITGA5, ITGB2, and ITGB5 top enrichment lists, including GO:0005201 (extracellular matrix structural constituent), GO:0005178 (integrin binding), GO:0019838 (growth factor binding), GO:0030020 (extracellular 

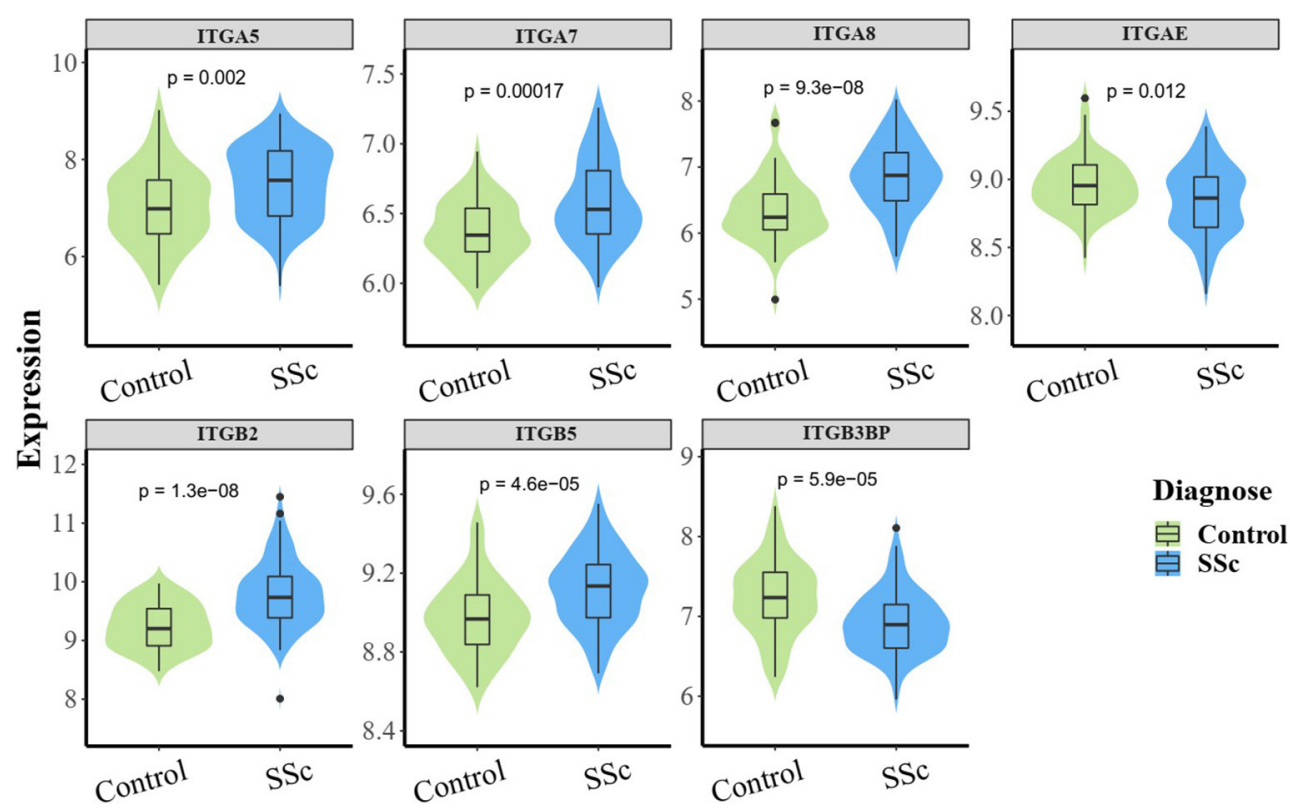

\section{Diagnose \\ 官 Control \\ 皇 SSc}

FIGURE 1 | The aberrant mRNA expression of integrin genes in SSc patients. Differentially expressed violin plot of integrin family genes in skin samples of patients with SSc (89 SSc vs. 61 normal control). SSc, systemic sclerosis.
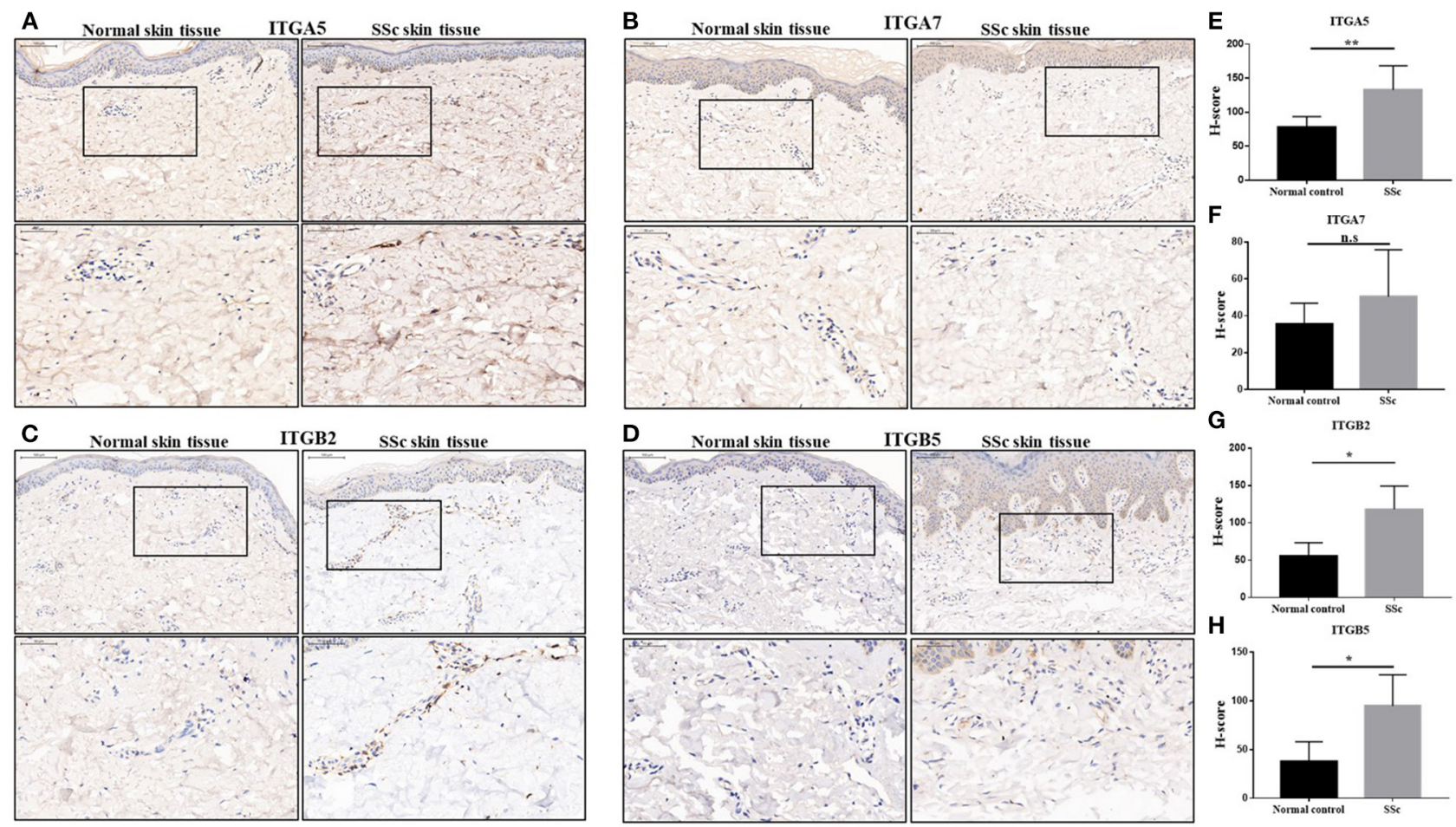

FIGURE 2 | The protein expressions of integrin $\alpha 5$, integrin $\alpha 7$, integrin $\beta 2$, and integrin $\beta 5$ in the skin tissues of SSc patients. (A-D) Representative images of integrin $\alpha 5$, integrin $\alpha 7$, integrin $\beta 2$, and integrin $\beta 5$ immunohistochemical staining in the skin tissues of SSc patients and normal controls. (E-H) Comparison of the $\mathrm{H}$-score values of integrin $\alpha 5$, integrin $\alpha 7$, integrin $\beta 2$, and integrin $\beta 5$ in SSc patients and normal control. The data are represented as mean \pm SD. Statistical differences were performed using the Mann-Whitney test. ${ }^{\star} p<0.05$, ${ }^{\star *} p<0.01$. SSc, systemic sclerosis. 
TABLE 2 | Demographic and clinical characteristics of subjects at skin biopsy.

\begin{tabular}{lccc}
\hline Characteristics & $\begin{array}{c}\text { SSc patients } \\
(\boldsymbol{n}=\mathbf{6 1})\end{array}$ & $\begin{array}{c}\text { Controls } \\
(\boldsymbol{n}=\mathbf{3 6})\end{array}$ & $\boldsymbol{p}$-value \\
\hline Age median (range), years & $54(22-82)$ & $\begin{array}{c}50 \\
(23-67)\end{array}$ & 0.025 \\
Gender, female/male, $n$ (\%) & $45 / 16(73.77)$ & $\begin{array}{c}29 / 7 \\
(80.56)\end{array}$ & 0.448 \\
Type of SSc, $\boldsymbol{n}$ (\%) & & & \\
dcSSc & & \\
IcSSC & $43(70.49)$ & \\
Total mRSS, median (range) & $18(29.51)$ & \\
Interstitial lung disease, $n$ (\%) & $14(2-39)$ & \\
FVC of predicted \%, median (range) & $76(37.70)$ & \\
DLCO of predicted \%, median (range) & $61(23-120)$ & \\
Antibody & & \\
Negative, $n$ (\%) & $17(27.87)$ & \\
RNP, $n$ (\%) & $3(4.92)$ & \\
ACA, $n$ (\%) & $7(11.48)$ & \\
ATA, $n$ (\%) & $17(27.87)$ & \\
RNAP, $n$ (\%) & $17(27.87)$ \\
Immunosuppressive agents, $n$ (\%) & $17(27.87)$ & \\
\hline
\end{tabular}

SSc, systemic sclerosis; dcSSc, diffused cutaneous systemic sclerosis; ICSSC, limited cutaneous systemic sclerosis; mRSS, modified Rodnan skin thickness score; FVC, forced vital capacity; DLCO, carbon monoxide breath diffusion capacity; RNP, anti-U1 RNP antibody; ACA, anti-centromere antibody; ATA, anti-topoisomerase antibody; RNAP, anti-RNA polymerase III antibody.

matrix structural constituent conferring tensile strength), and GO:0048407 (platelet-derived growth factor binding) in molecular function enrichment; GO:0062023 (collagencontaining extracellular matrix), GO:0031012 (extracellular matrix), GO:0005604 (basement membrane), GO:0005788 (endoplasmic reticulum lumen), GO:0005581 (collagen trimer), GO:0005925 (focal adhesion), and GO:0030055 (cell-substrate junction) in cellular components enrichment; GO:0030198 (ECM organization) and GO:0043062 (extracellular structure organization) in biological processes enrichment (Figures 5A-I); and hsa04510 (focal adhesion), hsa04512 (ECM-receptor interaction), hsa05144 (malaria), hsa04670 (leukocyte transendothelial migration), and hsa04974 (protein digestion and absorption) in KEGG enrichment (Figures 6A-C). In summary, ITGA5, ITGB2, and ITGB5 might synergistically promote SSc through the extracellular matrix turnover, ECM-receptor interaction, focal adhesion, and leukocyte trans-endothelial migration. It is worth mentioning that ITGA5- and ITGB5associated genes were enriched for GO:0001525 (angiogenesis) in the biological processes enrichment.

In addition, ITGA5-associated genes were uniquely enriched for GO:0007015 (actin filament organization), GO:0051493 (regulation of cytoskeleton organization), GO:0032956 (regulation of actin cytoskeleton organization), GO:0031589 (cell-substrate adhesion), GO:1902903 (regulation of supramolecular fiber organization), GO:0030335 (positive regulation of cell migration), and GO:0032970 (regulation of actin filament-based process). The results indicated that ITGA5 might be more involved in the organization and regulation of actin cytoskeleton, cell adhesion, and migration in SSc. ITGB2-associated genes were uniquely enriched for GO:0043299 (leukocyte degranulation), GO:0050900 (leukocyte migration), GO:0006909 (phagocytosis), GO:0002275 (myeloid cell activation involved in immune response), GO:0002444 (myeloid leukocyte mediated immunity), GO:0042110 (T-cell activation), GO:0042119 (neutrophil activation), and GO:0002250 (adaptive immune response). These results implied that ITGB2 might be important for inflammation by cytokine binding and activation of T cells, monocytes, and granulocytes in SSc. ITGB5 associated genes uniquely enriched for GO:0032963 (collagen metabolic process), GO:0030199 (collagen fibril organization), GO:0071559 (response to transforming growth factor beta), GO:0071560 (cellular response to transforming growth factor beta stimulus), GO:0007179 (transforming growth factor beta receptor signaling pathway), GO:0007178 (transmembrane receptor protein serine/threonine kinase signaling pathway), and GO:0032964 (collagen biosynthetic process). These results indicated that ITGB5 might be involved in the transforming growth factor beta signaling pathway.

\section{DISCUSSION}

Integrin plays important and complex roles in SSc, but integrintargeted therapy has not shown significant efficacy in SSc patients, which may be due to that the precise role of integrin is currently ambiguous and unsuitable option for treatment target. In this study, we used the GEO database to explore the expression and potential roles of integrin family genes in SSc skin, hoping to find a feasible target. We found that the mRNA and protein levels of ITGA5, ITGB2, and ITGB5 were abnormally overexpressed in the skin of SSc. Further analysis indicated that the mRNA levels of ITGA5, ITGB2, and ITGB5 were positively correlated with mRSS score. Besides, we also found that the mRNA levels of ITGB2 and ITGB5 were associated with positive autoantibodies. ITGA5, ITGB2, and ITGB5 showed strong correlations with various stromal cells including endothelial cell and fibroblast, respectively.

Mechanically, our results showed ITGA5, ITGB2, and ITGB5 were mainly enriched for ECM and cell-matrix interaction, indicating that they might promote SSc by affecting ECM turnover, ECM-receptor interaction, focal adhesion, and leukocyte trans-endothelial migration. Growth factor, chemokines, ADP, and thrombin can interact with talin and kindlin, called "inside-out" signaling, and promote the activity of integrins $(6,21)$. On the other hand, integrins, like integrin $\alpha 2 \beta 1$ (coded by ITGA2 and ITGB1), can directly bind to GFOGER-like motifs of collagen I to enhance collagen synthesis without the help of inside-out signaling (22). On the other hand, integrins bind to ECM ligands and subsequently trigger the accumulation of complex adaptors and signaling proteins, activating integrin downstream signaling pathways, such as the activation of focal adhesion kinase (FAK), SRC, AKT, and ERK pathways, which is known as the "outside-in" signaling, and consequently regulating cell behavior, such as the inhibition of cell death, the regulation 
A

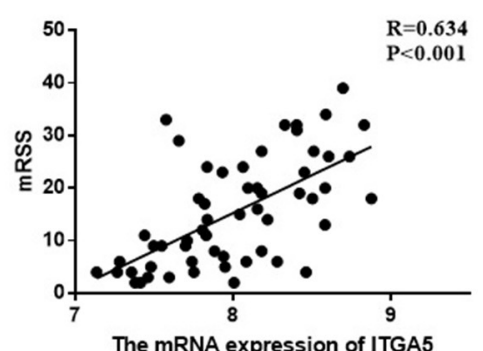

The mRNA expression of ITGA5

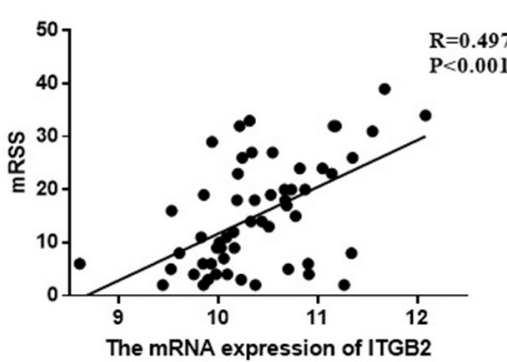

ITGB2

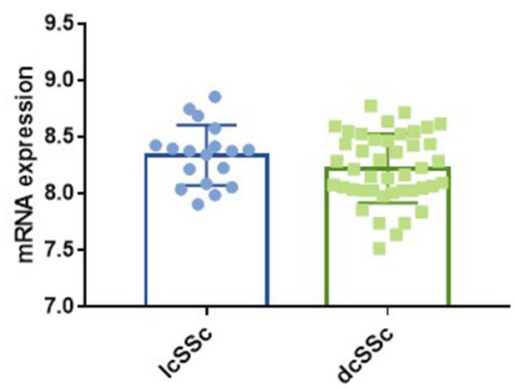

ITGB2

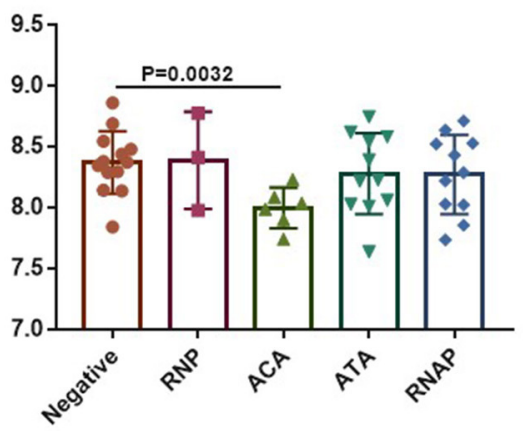

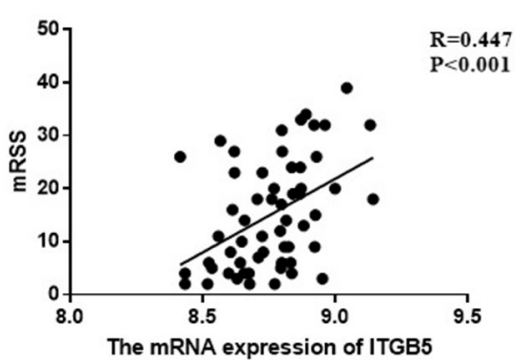

ITGB5

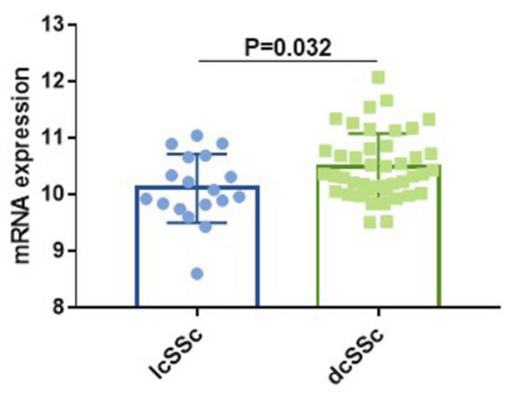

ITGB5
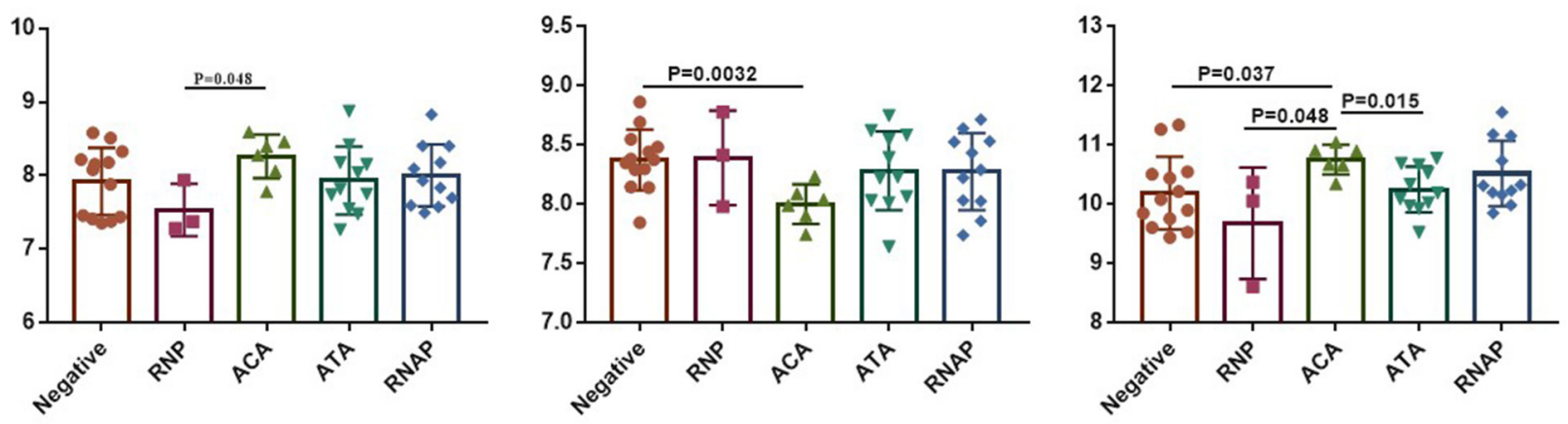

FIGURE 3 | Correlation between aberrant expressed integrin family genes and clinical features of SSc from GSE58095. (A) The mRNA expression of ITGA5, ITGB2, and ITGB5 genes was positively correlated with mRSS, respectively. (B) The mRNA expression levels of ITGA5 and ITGB5 were higher in dcSSc than in IcSSc. (C) ITGB2 was downregulated in patients seropositive for ACA than in lacking specific antibodies. The gene expression of ITGB5 was higher in patients seropositive for ACA than in those lacking specific antibodies or those seropositive for either RNP or ATA. SSc, systemic sclerosis; dcSSc, diffused cutaneous systemic sclerosis; IcSSc, limited cutaneous systemic sclerosis; mRSS, modified Rodnan skin thickness score; RNP, anti-U1 RNP antibody; ACA, anti-centromere antibody; ATA, anti-topoisomerase antibody; RNAP, anti-RNA polymerase III antibody.

of cytoskeletal dynamics and cellular structure, and intracellular transport or migration (23). In summary, these results indicated that as receptors of ECM components, ITGA5, ITGB2, and ITGB5 participate in the fibrosis process through connecting ECM components with FAK, which led to FAK activation, further promoting the proliferation, activation, and migration of fibroblasts and endothelial cells.

Our study also showed that ITGA5, ITGB2, and ITGB5 may mediate the migration of leukocytes from blood vessels to skin tissue by interacting with chemokines (CXCL12, CCL2) and vascular endothelial cell adhesion molecules
(JAM3/CDH5/ICAM 1/PECM1) and participate in the immune inflammatory process of SSc. Overexpression of ITGB2 can recognize ligands such as intercellular adhesion molecule 1 (ICAM-1) and vascular cell adhesion molecule 1 (VCAM-1) on the surface of endothelial cells, and then provide the direct cellular binding to vascular endothelial cells and fibroblasts, which facilitates the infiltration of inflammatory cells to the endothelium and subsequent transmigration (24). A recent study also showed that interleukin $1 \beta$ (IL-1 $\beta$ ) not only induced inflammatory but also increased the expression of integrin $\alpha 5 \beta 1$ to promote trans-endothelial migration of peripheral blood 

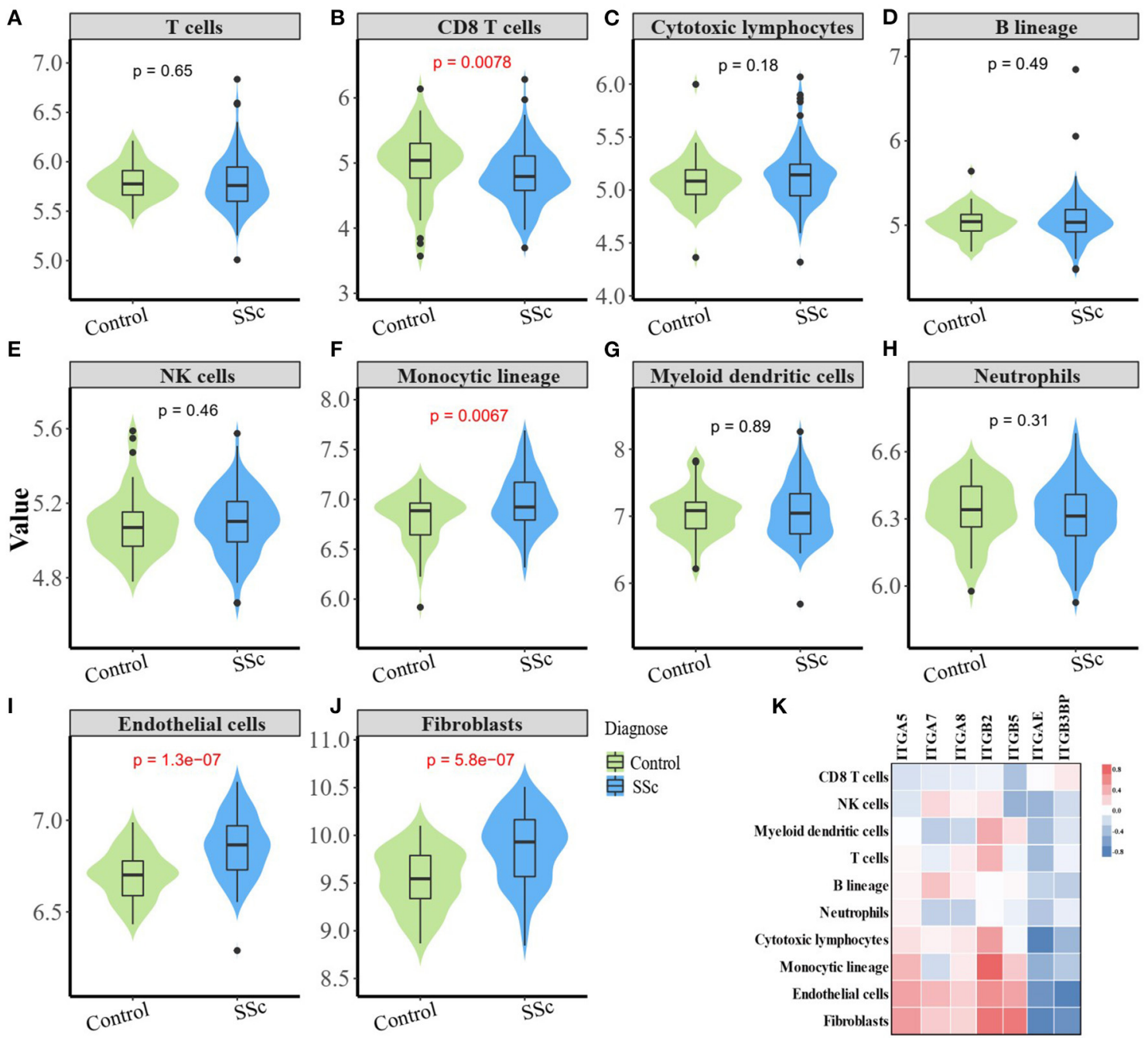

FIGURE 4 | Correlation between aberrant expressed integrin family gene and the infiltration of immune and stromal cells in the skin of SSc. (B) The abundance of $\mathrm{CD}^{+} \mathrm{T}$ cell is relatively low in the skin from SSc patients than healthy controls. (F, I, J) The abundance of monocytic lineage, endothelial, and fibroblast were higher in SSc patients compared with healthy control, respectively. (A, C-E, G, H) The abundance of total T cells, cytotoxic lymphocytes, B cells, NK cells, myeloid dendritic cells, and neutrophils showed no difference between SSc patients and healthy control. (K) The mRNA expression level of ITGA5 was positively correlated with fibroblasts $(r=0.47, p<0.0001)$, endothelial cells $(r=0.44, p<0.0001)$, and monocytes $(r=0.35, p<0.0001)$. The mRNA expression level of ITGB2 was significantly higher than that of fibroblasts $(r=0.64, p<0.0001)$, endothelial cells $(r=0.52, p<0.0001)$, T cells $(r=0.36, p<0.0001)$, and myeloid dendritic cells ( $r=0.39, p<0.0001)$. The mRNA expression level of ITGB5 was positively correlated with fibroblasts $(r=0.63, p<0.0001)$, endothelial cells $(r=0.43, p<0.0001)$, and monocytes $(r=0.26, p=0.0021)$. SSc, systemic sclerosis.

mononuclear cells (PBMC) in human brain microvascular endothelial cells (25). Generally speaking, the interaction between inflammatory cells, endothelial cells, and fibroblasts regulated by integrin family members including ITGA5, ITGB2, and ITGB5 may lead to the activation of vascular endothelial cells and fibroblasts in SSc. Our results indicated that ITGA5 and ITGB5 also synergistically affected angiogenesis and endothelial cell function. Wang et al. found that higher matrix stiffness increased VEGFR2 expression in human umbilical vein endothelial cells and that the integrin $\alpha V \beta 5 / A k t / S p 1$ pathway participated in stiffness-mediated effects on VEGFR2 upregulation (26). Integrin $\alpha 5 \beta 1$ levels in endothelial cells were induced in response to several angiogenic factor stimuli, such as IL-8, bFGF, or TNF $\alpha$. Upregulated integrin $\alpha 5 \beta 1$ participated in regulating angiogenesis by interacting with diverse partners such as VEGFR-1, CD97, and UPAR (27-29). In addition, blocking integrin $\alpha 5$ subunits by specific monoclonal antibodies or small peptides has become a potential strategy for anti-angiogenesis therapy (30).

Overall, integrin members have a compensatory and synergistic effect, which may explain why the current therapies targeting integrin have not achieved good efficacy in fibrosis or SSc. Combined therapy targeting multiple integrin members in SSc should be considered. 


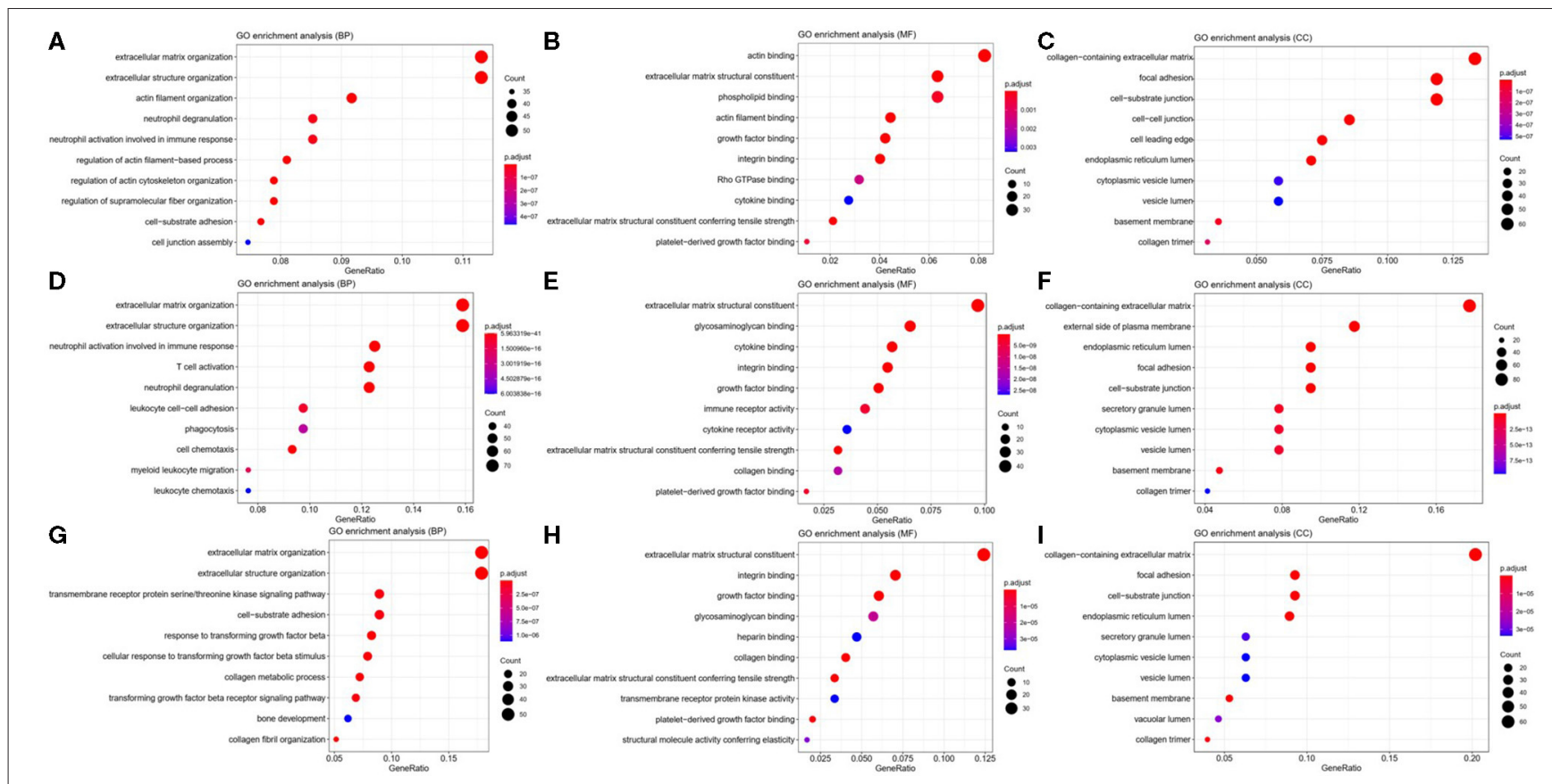

FIGURE 5 | Possible mechanisms of ITGA5, ITGB2, and ITGB5 involved in SSc. Top 10 significantly enriched GO terms for ITGA5 (A-C), ITGB2 (D-F), and ITGB5 (G-I). The size of the circle represents gene count. Different colors of circles represent different adjusted $p$-value. Gene ratio means the number of genes in /TGA5 and ITGB2/B5 co-expressed genes ( $r>0.5$ ); genes that belong to this pathway divided by the number of genes in the background gene cluster that belong to this pathway. GO, Gene Ontology; SSc, systemic sclerosis.

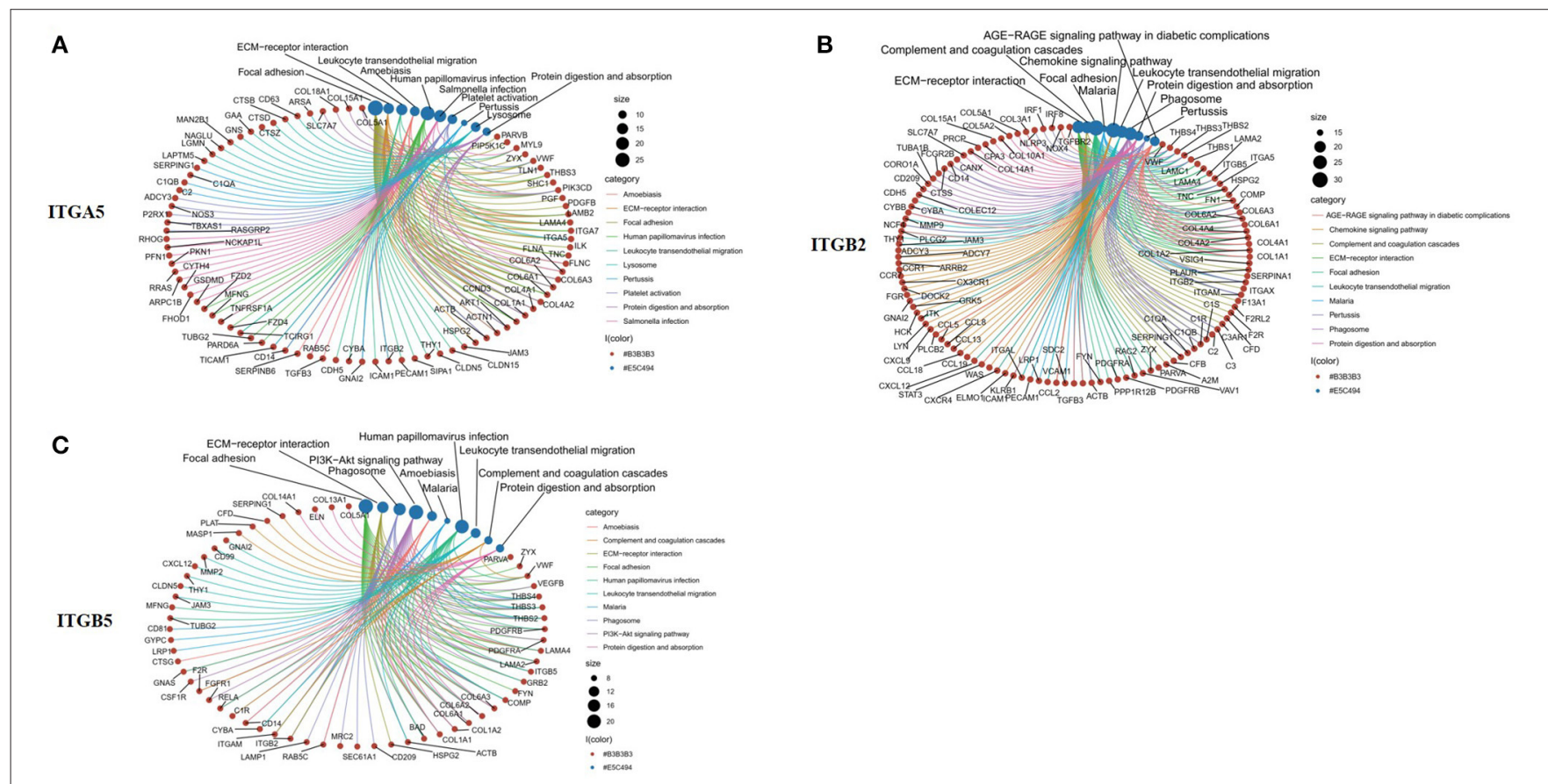

FIGURE 6 | Top 10 significantly enriched KEGG pathway for ITGA5, ITGB2, and ITGB5 involved in SSc. (A) ITGA5; (B) ITGB2; (C) ITGB5. The red circle represents genes, and the blue circle represents related signaling pathways enriched by genes. KEGG, Kyoto Encyclopedia of Genes and Genomes; SSc, systemic sclerosis. 
Our study also suggested that ITGA5, IGTB5, and IGTB2 participated in SSc pathogenesis in unique ways. RNA interference high-throughput assay showed that ITGA5 is one of the genes that affect the myofibroblast phenotype of SSc skin fibroblasts (31). A higher expression level of ITGA5 was found in the serum-derived fibroblasts of IPF patients than in the normal cells (32), which facilitated a more aggressive proliferative phenotype of fibroblasts (33). This was consistent with our findings that ITGA5 mRNA expression was positively correlated with fibroblasts, suggesting that ITGA5 may mainly affect the phenotype of fibroblasts and affect the process of fibrosis. We also found that ITGA5 was related with cytoskeleton and actin. Supportively, a previous study showed that ITGA5 can modulate peripheral actin organization (34). Presumably, ITGA5 participated in cell stiffness and contraction phenotype, while ITGB5 participated in TGF- $\beta$ signaling in SSc patients. ITGB5 combined with $\alpha \mathrm{V}$ can bind to arginine-glycine-aspartic acid (RGD) leading to the activation of TGF- $\beta 1$ and the enhancement of TGF- $\beta$ signaling by physical association with TGF- $\beta$ RII $(35,36)$. Yoshihide et al. found that $\alpha \mathrm{V} \beta 5$ was upregulated in scleroderma fibroblasts and that the transient overexpression of $\alpha \mathrm{V} \beta 5$ increased the human alpha2 (I) collagen gene expression in normal fibroblasts (37). ITGB2 correlated with activation of $\mathrm{T}$ cell, neutrophil, and monocyte, indicating that ITGB2 may be involved in the inflammation of SSc. Physiologically, ITGB2 was important for host defense, which was exclusively expressed in leukocytes promoting leukocyte adhesion and ensuing extravasation (38). In summary, each integrin has its specific role and function in SSc, which involves many aspects such as immunity, inflammation, and fibrosis, indicating that the integrin family members play a wide and important role. In addition, it also reminds us of the importance of further searching for molecules that can simultaneously regulate the activation of several integrin members such as talin and kindlins (6).

This study has some limitations. The protein levels of integrin $\alpha 8$, integrin $\alpha \mathrm{E}$, and integrin $\beta 3$ binding protein remain to be detected in the skin tissues of SSc patients. Second, our study only focused on the integrin mRNA expression in skin samples, leaving the expression and function of integrin in PBMC, lung, and other involved tissues unconsidered. Therefore, further

\section{REFERENCES}

1. Thannickal VJ, Zhou Y, Gaggar A, Duncan SR. Fibrosis: ultimate and proximate causes. J Clin Invest. (2014) 124:4673-7. doi: 10.1172/ JCI74368

2. Cipriani P, Marrelli A, Liakouli V, Di Benedetto P, Giacomelli R. Cellular players in angiogenesis during the course of systemic sclerosis. Autoimmun Rev. (2011) 10:641-6. doi: 10.1016/j.autrev.2011.04.016

3. Liakouli V, Cipriani P, Di Benedetto P, Ruscitti P, Carubbi F, Berardicurti $\mathrm{O}$, et al. The role of extracellular matrix components in angiogenesis and fibrosis: possible implication for Systemic Sclerosis. Mod Rheumatol. (2018) 28:922-32. doi: 10.1080/14397595.2018.1431004

4. Moreno-Layseca P, Icha J, Hamidi H, Ivaska J. Integrin trafficking in cells and tissues. Nat Cell Biol. (2019) 21:122-32. doi: 10.1038/s41556-0180223-z studies are necessary to validate our findings and conjectures in vitro and in vivo.

In conclusion, this study revealed that the mRNA and protein levels of ITGA5, IGTB5, and IGTB2 were upregulated in the skin tissue of SSc patients. ITGA5, IGTB5, and IGTB2 may participate in multiple pathological processes, and preliminary evidence of existing overlapping mechanisms has been noticed. Further studies are warranted.

\section{DATA AVAILABILITY STATEMENT}

Publicly available datasets were analyzed in this study. This data can be found here: https://www.ncbi.nlm.nih.gov/geo/ query/acc.cgi?acc=GSE58095 https://www.ncbi.nlm.nih.gov/ geo/query/acc.cgi?acc=GSE65336 https://www.ncbi.nlm.nih. gov/geo/query/acc.cgi? acc=GSE95065.

\section{ETHICS STATEMENT}

The studies involving human participants were reviewed and approved by the Ethical Committee of the Peking university people's hospital. The patients/participants provided their written informed consent to participate in this study.

\section{AUTHOR CONTRIBUTIONS}

RM designed this study. DX, TL, and RW contributed to the data interpretation and analysis. All authors contributed to the drafting and revising of the manuscript and have critically reviewed and approved the final submitted version to be published.

\section{FUNDING}

This work was supported by grants from the National Natural Science Foundation of China (No. 81771706).

\section{ACKNOWLEDGMENTS}

The gene expression datasets were obtained from the GEO database. We thank all of the investigators for sharing the data.

5. Campbell ID, Humphries MJ. Integrin structure, activation, and interactions. Cold Spring Harb Perspect Biol. (2011) 3:a004994. doi: 10.1101/cshperspect.a004994

6. Sun Z, Costell M, Fassler R. Integrin activation by talin, kindlin and mechanical forces. Nat Cell Biol. (2019) 21:2531. doi: 10.1038/s41556-018-0234-9

7. Nolte M, Margadant C. Controlling immunity and inflammation through integrin-dependent regulation of TGF-beta. Trends Cell Biol. (2020) 30:4959. doi: 10.1016/j.tcb.2019.10.002

8. Plow EF, Meller J, Byzova TV. Integrin function in vascular biology: a view from 2013. Curr Opin Hematol. (2014) 21:2417. doi: 10.1097/MOH.0000000000000042

9. Chen $\mathrm{C}$, Li R, Ross RS, Manso AM. Integrins and integrinrelated proteins in cardiac fibrosis. J Mol Cell Cardiol. (2016) 93:162-74. doi: 10.1016/j.yjmcc.2015.11.010 
10. Eliceiri BP. Integrin and growth factor receptor crosstalk. Circ Res. (2001) 89:1104-10. doi: 10.1161/hh2401. 101084

11. Sun Z, Guo SS, Fassler R. Integrin-mediated mechanotransduction. J Cell Biol. (2016) 215:445-56. doi: 10.1083/jcb.201609037

12. Bachmann M, Kukkurainen S, Hytonen VP, Wehrle-Haller B. Cell Adhesion by Integrins. Physiol Rev. (2019) 99:1655-99. doi: 10.1152/physrev.00036.2018

13. Luzina IG, Todd NW, Nacu N, Lockatell V, Choi J, Hummers LK, et al. Regulation of pulmonary inflammation and fibrosis through expression of integrins alphaVbeta 3 and alphaVbeta5 on pulmonary $\mathrm{T}$ lymphocytes. Arthritis Rheum. (2009) 60:1530-9. doi: 10.1002/art.24435

14. Chairta P, Nicolaou P, Christodoulou K. Genomic and genetic studies of systemic sclerosis: a systematic review. Hum Immunol. (2017) 78:15365. doi: 10.1016/j.humimm.2016.10.017

15. Gerber EE, Gallo EM, Fontana SC, Davis EC, Wigley FM, Huso DL, et al. Integrin-modulating therapy prevents fibrosis and autoimmunity in mouse models of scleroderma. Nature. (2013) 503:126-30. doi: 10.1038/nature12614

16. Henderson NC, Arnold TD, Katamura Y, Giacomini MM, Rodriguez JD, McCarty JH, et al. Targeting of alphav integrin identifies a core molecular pathway that regulates fibrosis in several organs. Nat Med. (2013) 19(12):1617-24. doi: 10.1038/nm.3282

17. Jensen K, Krusenstjerna-Hafstrom R, Lohse J, Petersen KH, Derand H. A novel quantitative immunohistochemistry method for precise protein measurements directly in formalin-fixed, paraffin-embedded specimens: analytical performance measuring HER2. Mod Pathol. (2017) 30:18093. doi: 10.1038/modpathol.2016.176

18. Becht E, Giraldo NA, Lacroix L, Buttard B, Elarouci N, Petitprez F, et al. Estimating the population abundance of tissue-infiltrating immune and stromal cell populations using gene expression. Genome Biol. (2016) 17:218. doi: 10.1186/s13059-016-1113-y

19. Assassi S, Swindell WR, Wu M, Tan FD, Khanna D, Furst DE, et al. Dissecting the heterogeneity of skin gene expression patterns in systemic sclerosis. Arthritis Rheumatol. (2015) 67:3016-26. doi: 10.1002/art.39289

20. Guo X, Higgs BW, Bay-Jensen AC, Karsdal MA, Yao Y, Roskos LK, et al. Suppression of $\mathrm{T}$ cell activation and collagen accumulation by an anti-IFNAR1 $\mathrm{mAb}$, anifrolumab, in adult patients with systemic sclerosis. J Invest Dermatol. (2015) 135:2402-9. doi: 10.1038/jid.2015.188

21. Munger JS, Sheppard D. Cross talk among TGF-beta signaling pathways, integrins, and the extracellular matrix. Cold Spring Harb Perspect Biol. (2011) 3:a005017. doi: 10.1101/cshperspect.a005017

22. Zeltz C, Gullberg D. The integrin-collagen connection-a glue for tissue repair? J Cell Sci. (2016) 129:653-64. doi: 10.1242/jcs.180992

23. Kechagia JZ, Ivaska J, Roca-Cusachs P. Integrins as biomechanical sensors of the microenvironment. Nat Rev Mol Cell Biol. (2019) 20:45773. doi: 10.1038/s41580-019-0134-2

24. Dashti N, Mahmoudi M, Gharibdoost F, Kavosi H, Rezaei R, Imeni V, et al. Evaluation of ITGB2 (CD18) and SELL (CD62L) genes expression and methylation of ITGB2 promoter region in patients with systemic sclerosis. Rheumatol Int. (2018) 38:489-98. doi: 10.1007/s00296-017-3915-y

25. Labus J, Woltje K, Stolte KN, Hackel S, Kim KS, Hildmann A, et al. IL-1beta promotes transendothelial migration of PBMCs by upregulation of the FN/alpha5betal signalling pathway in immortalised human brain microvascular endothelial cells. Exp Cell Res. (2018) 373:99-111. doi: 10.1016/j.yexcr.2018.10.002

26. Wang $\mathrm{Y}$, Zhang $\mathrm{X}$, Wang $\mathrm{W}$, Xing $\mathrm{X}, \mathrm{Wu} \mathrm{S}$, Dong $\mathrm{Y}$, et al. Integrin alphaVbeta5/Akt/Sp1 pathway participates in matrix stiffness-mediated effects on VEGFR2 upregulation in vascular endothelial cells. Am J Cancer Res. (2020) 10:2635-48.

27. Orecchia A, Lacal PM, Schietroma C, Morea V, Zambruno G, Failla CM. Vascular endothelial growth factor receptor-1 is deposited in the extracellular matrix by endothelial cells and is a ligand for the alpha 5 beta 1 integrin. J Cell Sci. (2003) 116:3479-89. doi: 10.1242/jcs.00673

28. Tjong WY, Lin HH. The role of the RGD motif in CD97/ADGRE5and EMR2/ADGRE2-modulated tumor angiogenesis. Biochem Biophys Res Commun. (2019) 520:243-9. doi: 10.1016/j.bbrc.2019.09.113

29. Tarui T, Andronicos N, Czekay RP, Mazar AP, Bdeir K, Parry GC, et al. Critical role of integrin alpha 5 beta 1 in urokinase (uPA)/urokinase receptor (uPAR, CD87) signaling. J Biol Chem. (2003) 278:2986372. doi: $10.1074 /$ jbc.M304694200

30. Ramakrishnan V, Bhaskar V, Law DA, Wong MH, DuBridge RB, Breinberg D, et al. Preclinical evaluation of an anti-alpha5beta1 integrin antibody as a novel anti-angiogenic agent. J Exp Ther Oncol. (2006) 5:273-86.

31. Chadli L, Sotthewes B, Li K, Andersen SN, Cahir-McFarland E, Cheung M, et al. Identification of regulators of the myofibroblast phenotype of primary dermal fibroblasts from early diffuse systemic sclerosis patients. Sci Rep. (2019) 9:4521. doi: 10.1038/s41598-019-41153-w

32. Epstein Shochet G, Brook E, Israeli-Shani L, Edelstein E, Shitrit D. Fibroblast paracrine TNF-alpha signaling elevates integrin A5 expression in idiopathic pulmonary fibrosis (IPF). Respir Res. (2017) 18:122. doi: 10.1186/s12931-017-0606-x

33. Shochet GE, Brook E, Bardenstein-Wald B, Grobe H, Edelstein E, Israeli-Shani L, et al. Integrin alpha-5 silencing leads to myofibroblastic differentiation in IPF-derived human lung fibroblasts. Ther $A d v$ Chronic Dis. (2020) 11:2040622320936023. doi: 10.1177/20406223209 36023

34. Oh MA, Kang ES, Lee SA, Lee EO, Kim YB, Kim SH, et al. PKCdelta and cofilin activation affects peripheral actin reorganization and cell-cell contact in cells expressing integrin alpha5 but not its tailless mutant. J Cell Sci. (2007) 120:2717-30. doi: $10.1242 /$ jcs.003566

35. Kim KK, Sheppard D, Chapman HA. TGF-betal Signaling and Tissue Fibrosis. Cold Spring Harb Perspect Biol. (2018) 10:a022293. doi: $10.1101 /$ cshperspect.a022293

36. Margadant C, Sonnenberg A. Integrin-TGF-beta crosstalk in fibrosis, cancer and wound healing. EMBO Rep. (2010) 11:97105. doi: $10.1038 /$ embor.2009.276

37. Asano Y, Ihn H, Yamane K, Jinnin M, Tamaki K. Increased expression of integrin alphavbeta5 induces the myofibroblastic differentiation of dermal fibroblasts. Am J Pathol. (2006) 168:499-510. doi: 10.2353/ajpath.2006.041306

38. Tan SM. The leucocyte beta2 (CD18) integrins: the structure, functional regulation and signalling properties. Biosci Rep. (2012) 32:241-69. doi: 10.1042/BSR20110101

Conflict of Interest: The authors declare that the research was conducted in the absence of any commercial or financial relationships that could be construed as a potential conflict of interest.

Copyright (c) $2021 \mathrm{Xu}, \mathrm{Li}$, Wang and $\mathrm{Mu}$. This is an open-access article distributed under the terms of the Creative Commons Attribution License (CC BY). The use, distribution or reproduction in other forums is permitted, provided the original author(s) and the copyright owner(s) are credited and that the original publication in this journal is cited, in accordance with accepted academic practice. No use, distribution or reproduction is permitted which does not comply with these terms. 